\title{
Research
}

\section{Three Years Experience of Bilateral Simultaneous Percutaneous Nephrolithotomy - A Single Center Study in Pakistan}

Shakeel Haseeb Uddin Siddique*, Muhammad Shoaib Mithani, Salman el Khalid, Fakhir Yousuf, Sunil Kumar, and Muhammad Saleem

The Kidney Centre, Post Graduate Training Institute, Karachi, Pakistan

${ }^{*}$ Correspondence to: Shakeel Haseeb Uddin Siddique; Registrar Urology, The Kidney Centre, Post Graduate Training Institute, Karachi, Pakistan; Tel: 03333658696; Email: shakeel_dmc@hotmail.com

Received: Nov 23 ${ }^{\text {rd }}, 2020$; Accepted: Dec 11 ${ }^{\text {th }}, 2020$; Published: Dec $14^{\text {th }}, 2020$

Citation: Uddin-Siddique SH, Mithani MS, el-Khalid S, Yousuf F, Kumar S, Saleem M. Three years experience of bilateral simultaneous percutaneous nephrolithotomy - a single center study in Pakistan. Urology Open A Open J. 2020; I(2): 31-34. doi: 10.33169/uro.UOAOJ-I-108

\section{ABSTRACT \\ Objective \\ To study the outcomes of simultaneous bilateral PCNLs in adult population and discussing therapeutical benefits and complications.}

\section{Design}

A Retrospective Study.

\section{Setting}

The Kidney Centre ( The Post Graduate Training Institute, Karachi).

\section{Duration of Study}

Three years from January 2017 to December 2019.

\section{Methodology}

A retrospective chart review at one of the large urology institute in Karachi was conducted. The aim was to document the demographics, operation dynamics, outcomes and complications of simultaneous bilateral PCNL in adult population. Record of 60 patients who underwent simultaneous bilateral PCNL between January 2017 and December 2019 in our hospital were evaluated. Preoperative, post-operative laboratory values, operative time, stone clearance, complications and hospital stay were recorded. SPSS IBM version 20 was used for data entry and analysis.

\section{Results}

The mean age was $40.0 \pm 15.1$ years. The mean hospital stay was 3.5 days and mean operative time was 144 minutes. The transfusion rate was observed $16.7 \%$ and infracostal approach was most frequently used approach.

\section{Conclusion}

Simultaneous bilateral PCNL is a safe, efficacious, and cost-effective option in cases of bilateral renal calculi and should be considered an acceptable treatment option for patients with bilateral stones due to low morbidity, overall short hospital stays, comparable stone free rates, cost effectiveness and early return-to-normal activity time.

Keywords: Obstructive uropathy; Simultaneous bilateral percutaneous nephrolithotomy; Urinary tract stones. 


\section{INTRODUCTION}

UTS (urinary tract stones) is a frequently reported condition at a urology clinic. ${ }^{1}$ The treatment of UTS has gained much recognition worldwide as being the most common urological disease. The incidence of UTS in European countries is about $12 \%$ in men as compared to $7 \%$ in women. ${ }^{2,3}$ Calculi in urinary tract can occur unilaterally or bilaterally, however serious complications like obstructive uropathy and renal failure can occur more in bilateral UTS owing to risk factors like metabolic conditions and obstructions in the urinary tract systems. Therefore, bilateral UTS require immediate surgical management. ${ }^{4}$

In the past couple of years, surgeons often used to operate side with major disease burden or side which was symptomatic either via open nephrolithotomy or percutaneous nephrolithotomy (PCNL). ${ }^{5}$ The first reported evidence of operating renal stones was made in 1976 when Fernström and Johansson prioritized PCNL over open technique. ${ }^{6}$ From past two decades PCNLs were favored over open surgeries for the treatment of complex renal stones or one with larger sizes. ${ }^{7}$ Two major guidelines are usually put into consideration while using percutaneous technique. The European Association of Urology guidelines suggest using PCNL for removal of renal stones $\geq 2 \mathrm{~cm}$ and stones at lower pole $\geq 1.5 \mathrm{~cm}$ and the American Urological Association (AUA) guidelines suggest PCNL as foremost treatment for staghorn calculi. ${ }^{8}$

The concern however shifted to patients presenting with large bilateral stones as treating UTS bilaterally represents a challenge. ${ }^{9,10}$ In this regard open surgeries and Unilateral PCNL technique proved to have lesser therapeutical benefits resulting in a longer duration at hospital, high chances of bleeding and anesthesia concerns, therefore surgical practice modified treatment of bilateral renal stones via bilateral PCNL simultaneously. ${ }^{5-7}$

We aim to provide evidence of the safety of simultaneous bilateral PCNL in case of bilateral renal stones to strengthen the scientific evidence data. To the best of our search limited data is published nationally and internationally with such variable. This study may add national data regarding such clinical situations in our part of the world to shape the national and international guidelines.

\section{MATERIALS AND METHODS}

The data of the patients who underwent simultaneous bilateral PCNL between January 2017 and December 2019 at the kidney Centre, Karachi, Pakistan was explored. This institute is a high volume Center dealing with UTS. In a month more than one thousand endourological surgeries are being performed including more than one hundred PCNL. Patient's data were collected via retrospective reviews of hospital records manually. Native ethical review board approval was taken prior to the study (reference \#66-URO-062018).

In this study, 60 patients were included. Urinalysis and urine culture, tests for serum creatinine and hemoglobin levels, abdominal and pelvic ultrasonography, and plain abdominal radiography were performed in all patients preoperatively. Intravenous urography and / or CT evaluation was performed in all patients. Number and location of access points, blood loss, stone clearance, and complications according to Clavian Dindo classification were recorded. The indication for PCNL was based on the size and type of stone and resistance to extracorporeal shock wave lithotripsy. Preoperative preparations of the patients with bilateral kidney stones were conducted in accordance with simultaneous bilateral PCNL. Intraoperative findings, operative time (from retrograde pyelogram to the end of the procedure), and outcomes were also recorded. In all patients' X-ray KUB or ultrasound was done on the first postoperative day to identify any residual fragments (defined as any residual calculus regardless of size). The Double-J stent was removed under cystoscopy guidance 4 weeks later. During hospitalization, all patients were given prophylactic antibiotics to minimize any urinary tract infections. Complete blood count and serum creatinine were checked again 24 hours after the procedure. All patients were followed up at the clinic after 2 weeks and serum creatinine were repeated.

During procedure, retrograde pyelogram was performed in the lithotomy position with $4 \mathrm{fr}$ ureteric catheter bilaterally and fixed with 16 Fr Foley catheter, and coded with different colored tape for identification of the left or right side then the patient was turned prone under endotracheal general anesthesia and access to the desired collecting system (intersection of major calices) was obtained using an $18 \mathrm{G}$ puncture needle under fluoroscopic guidance. The track was formed using serial metallic dilators with telescopic technique until $27 \mathrm{Fr}$ and an Ampltz sheath (30 Fr) was inserted. The stones were fragmented with a pneumatic lithoclast and removed piece-by-piece with stone forceps for chemical analysis. On table clearance was checked by fluoroscope. Patients with radiolucent stones and suspicious for residual stones underwent placement of an antegrade Double-J catheter (4.7 Fr). After removal of the working sheath, $22 \mathrm{fr}$ nephrostomy tube was inserted in cases having significant perioperative bleeding, the wound was closed with 2-0 prolene sutures to control any subcutaneous bleeding. All procedures were performed by the consultant urologists and senior registrars. Simultaneous contralateral PCNL was performed after a reassessment of the anesthetist. Nephrostomy and Foley catheter were removed on the first Post-operative day. Most of the patients were discharged on second post-operative day. All patients with residual fragments underwent URS (ureterorenoscopy) or ESWL (extracorporeal shock wave lithotripsy ) according to residual stone size and position of stone.

\section{STATISTICAL ANALYSIS}

SPSS IBM version 20 was used for data entry and analysis. Descriptive statistics were used, Mean \pm S.D were obtained for continuous variable while $\mathrm{n}(\%)$ was observed for categorical data.

\section{RESULTS}

Out of 60 cases in our study 25 were female and 35 were male. The mean age was $40 \pm 15.1$ years with minimum was 17 and maximum age was 76 years (Table 1). A rise in creatinine level was observed in 14 cases from 1.3 to 1.9 but it was found a transient rise because when repeated after 2 weeks all levels recovered to base line normal values. Complete Stone clearance was observed in 52 cases and only 08 patients underwent ancillary procedures for removal of residual stone. Infracostal approach was the most frequent approach used for surgery (Table 2). In only 08 cases grade I complications were observed including fever (in 05 patients) and urinary leakage (in 03 patients) from nephrostomy site which were managed with dressing and urostomy bag application. There was no thoracic complication in supracostal approach and no secondary PCNL needed. 
Table 1. Demographic details and Clinical parameters of patients.

\begin{tabular}{|l|l|l|l|}
\hline VARIABLE & MEAN \pm S.D & $\begin{array}{l}\text { MINIMUM } \\
\text { VALUE }\end{array}$ & $\begin{array}{l}\text { MAXIMUM } \\
\text { VALUE }\end{array}$ \\
\hline AGE (YEARS) & $40 \pm 15.1$ & 17 & 76 \\
\hline BMI & $22.7 \pm 4.5$ & 14 & 33 \\
\hline SIZE OF STONE IN RIGHT KIDNEY(cm) & $2.4 \pm 0.8$ & 1.0 & 4.0 \\
\hline SIZE OF STONE IN LEFT KIDNEY (cm) & $2.2 \pm 0.9$ & 1.0 & 5.0 \\
\hline HOSPITAL STAY (days) & $3.5 \pm 0.7$ & 3.0 & 6.0 \\
\hline OPERATIVE TIME (minutes) & $144.9 \pm 31$ & 90 & 210 \\
\hline PRE OPERATIVE CREATININE(mg/dl) & $0.96 \pm 0.1$ & 0.5 & 1.3 \\
\hline POST OPERATIVE CREATININE (mg/dl) & $1.1 \pm 0.2$ & 0.7 & 1.9 \\
\hline $\begin{array}{l}\text { CREATININE AT 15TH DAY FOLLOW UP } \\
\text { (mg/dl) }\end{array}$ & $0.94 \pm 0.17$ & 0.4 & 1.2 \\
\hline PRE OPERATIVE HEMOGLOBIN $(\mathrm{gm} / \mathrm{dl})$ & $13.7 \pm 2.1$ & 10.8 & 17.7 \\
\hline POST OPERATIVE HEMOGLOBIN $(\mathrm{gm} / \mathrm{dl})$ & $11.0 \pm 2.1$ & 7.2 & 15.0 \\
\hline
\end{tabular}

Table 2. Categorical variables of patients.

\begin{tabular}{|c|c|c|}
\hline \multirow{3}{*}{\begin{tabular}{|l} 
Variable \\
Gender
\end{tabular}} & \multicolumn{2}{|c|}{ N (\%) } \\
\hline & Male & $35(58.6)$ \\
\hline & Female & $25(41.7)$ \\
\hline \multirow{3}{*}{ Approach } & Supracostal & $05(8.3)$ \\
\hline & Infracostal & $38(63.3)$ \\
\hline & Both & $17(28.3)$ \\
\hline \multirow{2}{*}{ Stone clearance } & Complete & $52(86.7)$ \\
\hline & Incomplete & $08(13.3)$ \\
\hline \multirow{3}{*}{ Ancillary procedure } & ESWL & $08(13.3)$ \\
\hline & URS & $02(3.3)$ \\
\hline & $2{ }^{\circledR} \mathrm{PCNL}$ & $00(0)$ \\
\hline \multirow{2}{*}{ No of stones } & Single & $29(48.3)$ \\
\hline & Multiple & $31(51.7)$ \\
\hline Blood transfusion & & $10(16.7)$ \\
\hline Post-operative creatinine $>1.2$ & & $14(23.3)$ \\
\hline Nephrostomy & & $20(33.3)$ \\
\hline \multirow{2}{*}{ complications } & Fever & $05(8.3)$ \\
\hline & Urinary leakage & $03(05)$ \\
\hline
\end{tabular}

\section{DISCUSSION}

Kidney stone comprise a serious worldwide health concern. An untreated renal stone may cause severe sepsis and may lead to damage of the kidney. Therefore, its removal is the main goal of urologist to relive any obstruction, prevention of recurrence of stone, to eradicate any associated infection. ${ }^{11}$ Our region Pakistan is situated in afro Asian stone belt (stretching from Egypt, middle east, Iran, Pakistan, India, Thailand up to Indonesia and the Philippines) and has consistently reported a high incidence of urolithiasis. ${ }^{12}$

When stone is present in both kidneys, it makes this goal, a challenge to urologist. If PCNL is indicated, it can be proceeded on both side simultaneously. Previously bilateral renal stones were treated with staged PCNL by treating single kidney at one operation. Several authors have shared their experiences regarding simultaneous bilateral PCNL but very limited local data available with such variables. Colon et al reported simultaneous bilateral PCNL for the first time in $1987 .{ }^{13}$ After that many surgeons also reported good results of simultaneous bilateral PCNL instead of single staged PCNL. ${ }^{14}$

In literature only limited scientific data is available regarding simultaneous bilateral PCNL or regarding proceeding for the second side PCNL simultaneously after completion of first side. Ugras et al reported drop in hemoglobin intraoperatively as a criterion to proceed for second side. ${ }^{15}$ Besides hemoglobin other important factors that should be considered before proceeding to the next side PCNL are prolonged operative time, hemodynamic instability and metabolic abnormalities like hyponatremia identified during the procedure on intraoperative laboratory test. If all these parameters allow than to proceed for the second side PCNL. ${ }^{16}$ In our study contralateral PCNL was done when the first side PCNL was completed and discussion with the anesthetist regarding hemodynamic stability of the patient.

Which side to operate first is another important question. Some urologist selects to treat more stone burden first and others prefer to treat small stone size first. Some urologist prefers to treat more symptomatic stone first. Obstructed kidney should be the priority. In selecting obstructive side first has an advantage to save the obstructive kidney in case if other side needs staged PCNL at the next surgery. In our study similar criteria was followed like to treat obstructed or more symptomatic kidney to treat first.

The possible concerns to attempt simultaneous bilateral PCNL is excessive blood loss during the procedure and the need for blood transfusion. Multiple studies reported different results for blood transfusion from 4 percent to $28 \%$ which is also comparable with our study $(16.7 \%) .{ }^{14,17,18}$

Besides transfusion and bleeding, Troxel and Low showed the complication rate of $26 \%$ fever in their study which is the most common complication of PCNL but observed in less numbers $(8.3 \%)$ in our study. ${ }^{19}$

Desai and colleagues also reported a similar study of simultaneous bilateral PCNL in 45 patients with similar parameters like mean hospital stay of ( 3.5 days' vs 6 days), but higher rate of secondary PCNL $(13 \%$ vs $0 \%) .{ }^{13}$

In another series reported by Wang et al who reported a randomized trial simultaneous bilateral PCNL with staged PCNL in 50 patients and 48 patients respectively, he reported prolonged operative duration of $269 \mathrm{~min}$ and he did not mention the parameter for secondary PCNL in his series. But stone clearance in both studies is comparable with the rate of $77 \%$ vs $86.7 \%$ in our series. Besides these no significant complications noted in the study like hydrothorax reported in his studies. $^{20}$

The important concern of deterioration of renal functions after simultaneous bilateral PCNL as increase in post-operative creatinine levels is one of the parameters in our study while not reported in many studies. In our study significant renal functions deterioration occurs immediately after procedure however this change was found temporarily and when renal functions were repeated in follow up clinical visit there was no significant difference in serum creatinine values as compared to the baseline values. Similar results were also reported in some other studies showing the safety of simultaneous procedure. ${ }^{13,18}$

In view of all these parameters simultaneous bilateral PCNL should be considered as a safe and feasible option for a surgeon by which the cost of one procedure can be saved including laboratory work up for staged PCNL, anesthesia and drugs can also be saved, it also includes only single hospital stay as also reported by KuKreja et al in his study. ${ }^{21}$ 
In the view of the above discussion, this study proved the modern day technique of simultaneous bilateral PCNL is safe and cost effective. There is no objective reason to hold back the decision of performing staged PCNL in bilateral renal stones until indicated.

\section{LIMITATIONS OF THE STUDY}

The evident limitation of the study is the retrospective nature of data. Larger sample size may give better representation in future trials. The pediatric population may also be included in future studies.

\section{CONCLUSION}

This study based on a single Centre experience from a developing country; Pakistan reveals that simultaneous bilateral PCNL is a safe and costeffective option in cases of bilateral renal calculi. It is associated with low morbidity, shorter hospital stays, high stone free rates, and early return-to-normal activity. Therefore, simultaneous bilateral PCNL should be considered an acceptable treatment option for patients with bilateral stone burden warranting percutaneous management. Further treatment protocols and guidelines can be formed by similar studies conducted prospectively.

\section{CONFLICTS OF INTEREST}

None.

\section{REFERENCES}

1. Leslie SW, Bhimji SS. StatPearls. Treasure Island FL: StatPearls Publishing LLC; 2017. Calculi, Renal.

2. Raheem OA, Khandwala YS, Sur RL, Ghani KR, Denstedt JD. Burden of Urolithiasis: Trends in Prevalence, Treatments, and Costs. Eur Urol Focus. 2017; 3(1): 18-26. doi: 10.1016/j.euf.2017.04.001

3. Arrabal-Martín M, Cano-García MC, Arrabal-Polo MÁ, et al. Etiopathogenic factors of the different types of urinary litiasis. Archivos espanoles de urologia. 2017; 70(1): 40-50

4. Stoller ML, Bolton DM. Urinary stone disease. In: Tanagho Emil A, McAninch Jack W., editors. Smiths General Urology. 15th editin. McGraw-Hill. Middle East edition; Lebanon: 2000. pp. 291-320.

5. Lingeman JE, Lifshitz DA, Evan AP. Surgical management of urinary lithiasis. Campblle Urology. 8th ed. Philadelphia-Pennsylvania: Saunders. 2002; 4: 3362-3396.

6. Al-Kohlany KM, Shokeir AA, Mosbah A, et al. Treatment of complete staghorn stones: A prospective randomized comparison of open surgery versus percutaneous nephrolithotomy. J Urol. 2005; 173(2): 469-473. doi: 10.1097/01.ju.0000150519.49495.88

7. Holman E, Khan AM, Pasztor I, Tóth C. Simultaneous bilateral compared with unilateral percutaneous nephrolithotomy. BJU Int. 2002; 89: 334-338. doi: 10.1046/j.1464- 4096.2001.01521.x

8. Pietrow PK, Auge BK, Lallas CD, Santa-Cruz RW, Newman GE,
Albala DM, et al. Pain after percutaneous nephrolithotomy: Impact of nephrostomy tube size. J Endourol. 2003; 17: 411-414. doi: $10.1089 / 089277903767923218$

9. Türk C, Knoll T, Petrik A, Sarica K, Straub M, Seitz C. Guidelines on urolithiasis. European Association of Urology Web site. http://uroweb. org/wp-content/uploads/22- Urolithiasis_LR.pdf. Updated 2014.

10. Preminger GM, Assimos DG, Lingeman JE, et al. Chapter 1: AUA guideline on management of staghorn calculi: diagnosis and treatment recommendations. J Urol. 2005; 173: 1991-2000. doi: 10.1097/01. ju.0000161171.67806.2a

11. Koga S, Arakaki Y, Matsuoka M. Staghorn calculi- long-term results of management. Br J Urol. 1999. 68: 122-129

12. Rizvi SA, Naqvi SA, Hussain Z, et al. The management of stone disease. BTU int. 2002; 89: 62-68

13. Colon-Perez B, Canto RJ, Ramos ME. Simultaneous bilateral nephrostolithotomies: Immediate results in three cases. J Endourol. 1987; 1: 209

14. Ahlawat R, Banerjee GK, Dalela D. Bilateral simultaneous percutaneous nephrolithotomy. A prospective feasibility study. Eur Urol.1995. 28: $116-118$

15. Ugras MY, Gedik E, Gunes A, Yanik M, Soylu A, Baydinc C. Some criteria to attempt second side safely in planned bilateral simultaneous percutaneous nephrolithotomy. Urology. 2008; 72: 996-1000. doi: 10.1016/j.urology.2008.08.002

16. Choi M, Brusky J, Weaver J, Amantia M, Bellman GC. Randomized trial comparing modified tubeless percutaneous nephrolithotomy with tailed stent with percutaneous nephrostomy with small-bore tube. J Endourol. 2006; 20: 766-770. doi: 10.1089/end.2006.20.766

17. Silverstein AD1, Terranova SA, Auge BK, Weizer. Bilateral renal calculi: Assessment of staged v synchronous percutaneous nephrolithotomy. JEndourol. 2004; 18(2): 145-151. doi: 10.1089/089277904322959770

18. Dushinski JW, Lingeman JE. Simultaneous bilateral percutaneous nephrolithotomy. J Urol. 1997. 158: 2065-2068.

19. Troxel SA, Low RK. Renal intrapelvic pressure during percutaneous nephrolithotomy and its correlation with the development of postoperative fever. J Urol. 2002. 168: 1348-1351. doi: 10.1097/01. ju.0000030996.64339.f1

20. Wang CJ, Chang CH, Huang SW. Simultaneous bilateral tubeless percutaneous nephrolithotomy of staghorn stones: A prospective randomized controlled study. Urol Res. 2011; 39(4): 289-294. doi: 10.1007/ s00240-010-0342-X

21. Kukreja R, Desai M, Patel S, Bapat S, Desai M. Factors affecting blood loss during percutaneous nephrolithotomy: Prospective study. J Endourol 2004; 18: 715-722. doi: 10.1089/end.2004.18.715 